\title{
Characterization of in-situ Doped Polycrystalline Silicon Using Schottky Diodes and Admittance Spectroscopy
}

\author{
H. Ayed ${ }^{1, *}$, L. Béchir ${ }^{1}$, M. Benabdesslem ${ }^{1}$, N. Benslim ${ }^{1}$, L. Mahdjoubi ${ }^{1}$, \\ T. Mohammed-Brahim², A. Hafdallah ${ }^{3}$, M.S. Aida ${ }^{3}$ \\ ${ }^{1}$ Laboratoire Cristaux et Couches Minces, Faculté des Sciences, Dpt de Physique 1(LESIMS), Université Badji \\ Mokhtar BP.12 Annaba, 23000, Algérie \\ ${ }^{2}$ GM-IETR, Université RENNES I, 35042 Rennes Cedex, France \\ ${ }^{3}$ Laboratory of Thin Films and Interfaces, Faculty of Science, Department of Physics, University of Constantine 1, \\ 25000 Algeria
}

(Received 28 July 2015; published online 15 March 2016)

\begin{abstract}
In this work, Schottky Au-Polycrystalline silicon diodes are successfully realised. The barrier height is around $\Phi_{\mathrm{B}}=0.74 \mathrm{eV}$ as determined from Capacitance - Bias (C-V) characteristics. The depth profile of the apparent doping is deduced from these measurements. Its behaviour leads to the experimental profile. Moreover, the diode admittance measurements versus the frequency and the temperature at different biases show the possibility to use this device to characterise the electrical quality of the polycrystalline silicon.
\end{abstract}

Keywords: Schottky Diodes, Characterisation, Polycrystalline silicon, Admittance Spectroscopy.

PACS numbers: 85.30.Hi, 85.30.Kk

\section{INTRODUCTION}

Polycrystalline silicon (polysilicon) has been an important material in integrated circuit technology for the last decades [1] is widely studied and applied now in different fields where the need of silicon devices made at low temperature is crucial. The most common example is the active matrix flat panel displays that uses polysilicon thin film transistors TFT' s made directly on glass substrate [2-3] has shown its interest in various applications, such as the solar cells [3]. In the photovoltaic field also, many woks were devoted to this material [4]. These major applications both need a lowtemperature deposition process $\left(<600{ }^{\circ} \mathrm{C}\right)$ due to the use of glass substrates and a high-quality polysilicon. It was shown that high-quality polysilicon can only be obtained from the crystallisation of amorphous deposited films [5]. It is usually columnar with grain boundaries perpendicular to the film surface. Consequently, the electrical transport properties may be different in the parallel and perpendicular to the surface directions. Most of characterisation techniques of polysilicon lead to the knowledge of the properties perpendicular to the surface of which some of them may be obtained by the characterisation of polycrystalline silicon Schottky diodes.

Here, Au-Polysilicon Schottky diodes are successfully performed and used to characterise the material [6-7]. Even if this device was previously used to study amorphous silicon [8-9], no known similar work was devoted to polysilicon.

\section{EXPERIMENTAL DETAILS}

Amorphous silicon films were deposited on 2 inches diameter $\mathrm{N}$ highly doped silicon wafers $(5 \mathrm{~m} \Omega . \mathrm{cm})$ by Low Pressure Chemical Vapor Deposition (LPCVD) ) in an horizontal tubular hot-wall reactor where the base pressure is $3 \times 10^{-5} \mathrm{~Pa}$. The deposition temperature was fixed at $550^{\circ} \mathrm{C}$. The films are amorphous in-situ doped, using silane and phosphine diluted in helium. Several phosphorus concentrations were obtained by varying the phosphine/silane gas flow ratio. The thickness of the layers is typically $2 \mu \mathrm{m}$. The, these films were crystallised at $600{ }^{\circ} \mathrm{C}$ under vacuum conditions (pressure lower than $3 \times 10^{-5} \mathrm{~Pa}$ ).

The Schottky diodes are then completed by thermal deposition of gold dots of $2.5 \mathrm{~mm}$ in diameter and $1000 \AA$ thickness. The current-voltage $(I-V)$ and capacitance-voltage $(C-V)$ characteristics at different temperature values $(T)$ were undertaken. The $1 / C^{2}$ vs $V$ relations of abrupt junction were to determine the doping concentration and the barrier height value. The conductance $(G)$ and capacitance $(C)$ vs temperature $(T)$ curves were also plotted at different frequency values (f). Also, the $G(f)$ and $C(f)$ plots at different values of regulated temperatures were performed. These characteristics constitute the basic measurement of the admittance spectroscopy technique that serves to characterize the deep traps of majority carriers when a metalsemiconductor is used. The admittance measurements were made at different frequencies between $100 \mathrm{~Hz}$ and $13 \mathrm{MHz}$ with a HP (4192A, $5 \mathrm{~Hz}-13 \mathrm{MHz})$ impedance analyzer. A liquid nitrogen cryostat is use to perform these measurements under high vacuum and to set the temperature with a precision better than $0.1{ }^{\circ} \mathrm{C}$.

\section{RESULTS}

\subsection{Preliminary Characterisation of Schottky Diodes}

When a nearly ideal SBDs is considered, it is assumed that the forward bias current of the device is due to thermionic emission current and it can be expressed as [10-12].

\footnotetext{
*ayedhami@yahoo.fr
} 


$$
I=I_{S}\left[\exp \left(\frac{q V}{n k T}\right)-1\right],
$$

where

$$
I_{S}=A^{*} S \mathrm{~T}^{2} \exp \left(\frac{-q \Phi_{B}}{k \mathrm{~T}}\right)
$$

is the saturation current, $\Phi_{\mathrm{B}}$ is the effective barrier height at zero bias, $A^{*}$ is the Richardson constant and equals to $112 \mathrm{~A} / \mathrm{cm}^{2} \mathrm{~K}^{2}$ for $n$-type $\mathrm{Si}$ [13], where $q$ is the electron charge, $V$ is the forward bias voltage, $S$ is the effective diode area, $k$ is the Boltzmann's constant, $T$ is the temperature in Kelvin, $\mathrm{n}$ is the ideality factor, and it is determined from the slope of the linear region of the forward bias $\ln I$ - $V$ characteristic through the relation:

$$
n=\frac{q}{k T} \frac{\partial V}{\partial(\ln I)}
$$

The Barrier height $\Phi_{\mathrm{B}}$ of the Schottky contact can be expressed by the followed equation [13]:

$$
\varphi_{B}=\frac{n k T}{q} \ln \left(\frac{A^{*} S T^{2}}{I_{S}}\right)
$$

Fig. 1 shows the direct and reverse $I-V$ characteristics at room temperature.The values of the $n$ and $\Phi_{B}$ obtained from $I-V$ characteristics using Eqs. (3) and (4) are 1.43 and $0.74 \mathrm{eV}$ respectively. The rectifying ratio at $+/-1 \mathrm{~V}$ is greater than $10^{4}$.

In Fig. 2 we show the $1 / C^{2}-V$ characteristics of $\mathrm{Au}-\mathrm{Si}$ diodes. Far from the interface, the value of built-in voltage $V_{b i}$ and the concentration of the donors $N_{D}$ are calculated by the assumption and the slope of the plot $1 / C^{2}$ versus $V$ :

$$
\frac{1}{C^{2}}=\frac{2}{q \varepsilon_{S} N_{d}}\left(V_{b i}-V\right)
$$

where $C$ is the capacitance, and $\varepsilon_{S}$ is the permittivity of semiconductor, $N_{D}$ is the doping concentration, and $V_{b i}$ is the built-in voltage. The intersection of this line with the axis of the tensions determines the diffusion potential $V=V_{b i}$. Similarly, the slope $\left(P_{C}\right)$ of the right $C^{-2}=$ $f(V)$ and the derivative $P_{C}=d C^{-2} / d V=2 /\left(q \varepsilon_{s} N_{d}\right)$ from the equation (5) give the $\mathrm{N}_{\mathrm{d}}$ concentration.

Using the following expression:

$$
N_{d}=\frac{-2}{q \varepsilon_{S} S^{2} P_{C}}
$$

The value of doping concentration $N_{d}=4.9 \times 10^{17} \mathrm{~cm}^{-3}$ and a potential diffusion of $1.3 \mathrm{eV}$ are obtained.

The decrease of $N_{d}$ at the interface can be due to the diffusion of tow sorts of compensator. The first type may be oxygen and the second may be the metallic atoms of the contact. In fact, Au is well known to be accepted for Si. Its diffusion towards the Si volume could provocate such compensation. However, this situation is not more probable than the oxygen compensation since this element, being very reactive with $\mathrm{Si}$, diffuses very slowly to the depth of the film and it is stoped at the interface to create the classical $\mathrm{SiO}_{2}$.

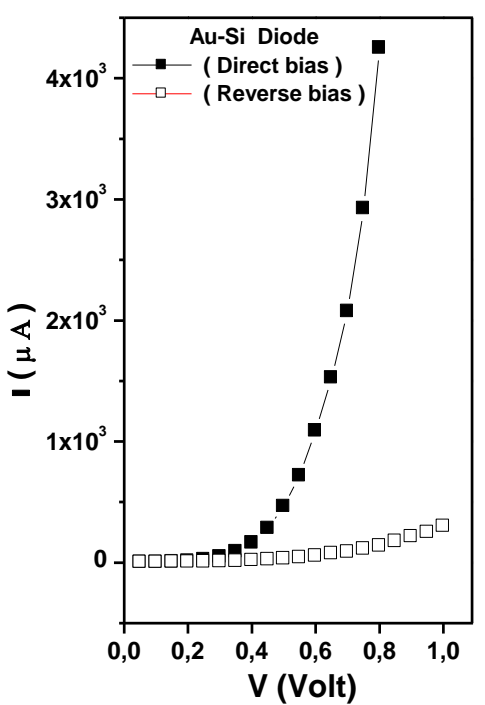

Fig. 1 - Direct and reverse $I$ - $V$ plots of Au-Si diode

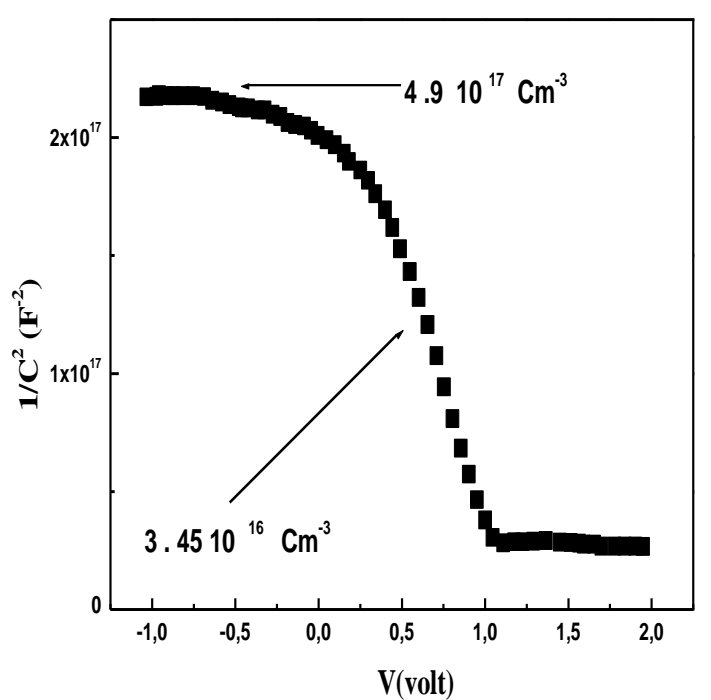

Fig. $2-\left(1 / C^{2}-V\right)$ characteristics of diode

\subsection{Admittance Spectroscopy}

As described with more details in a previous work [14-15], the set up of the admittance spectroscopy (AS) technique consists to record the diode conductance $(G)$ versus temperature $(T)$ for a given value of the measurement frequency. The presence of a trap is indicated by a peak of the conductance $G$ (or a point of inflection of the device capacitance $(C)$ which appears at a certain temperature $\left(T_{m}\right)$ [15] for a given value of the pulsation $\left(\omega_{i}\right)$ of the bridge conductance-meter. At the peak temperature $\left(T_{m}\right)$, the trap emission rate $\left(e_{i}\right)$ is equal to $\left(\omega_{i}\right)$ in the case of high level of signal modulation. For different values of $\omega$, a series of couples $\left(e_{i}, T_{m i}\right)$ is determined to plot the Arrhenius diagrams, $e_{i} / T_{m i}{ }^{2}$ as a function of $1 / T_{m i}$, leading to the knowledge of its energy position $E_{t}$ relatively to the intrinsic bands and the capture cross section of the trap $(\sigma)$. The peak height is used to determine its concentration $N_{t}$. Fig. 3 shows typical plots of G-T and C-T measurements of a Schottky Au-polycrystalline $\mathrm{Si}$ diode at a frequency $(f)$ of $20 \mathrm{kHz}$. The same spectroscopy is performed when $G(f)$ 


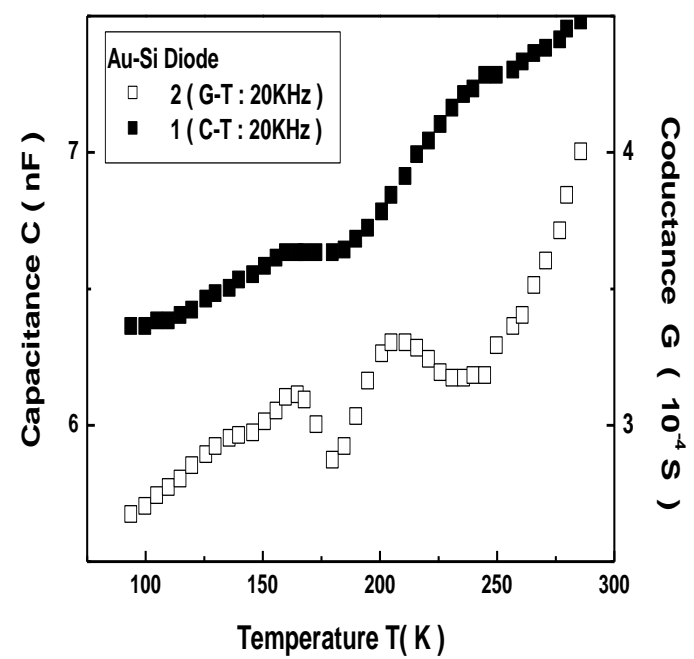

Fig. 3 - Typical plots of the conductance $G$ and the capacitance $C$ versus the temperature

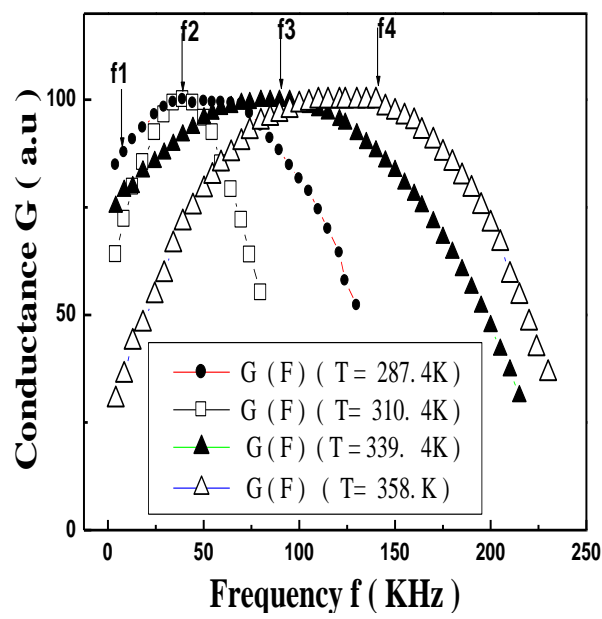

Fig. 4 - Typical G-F plots at different temperatures

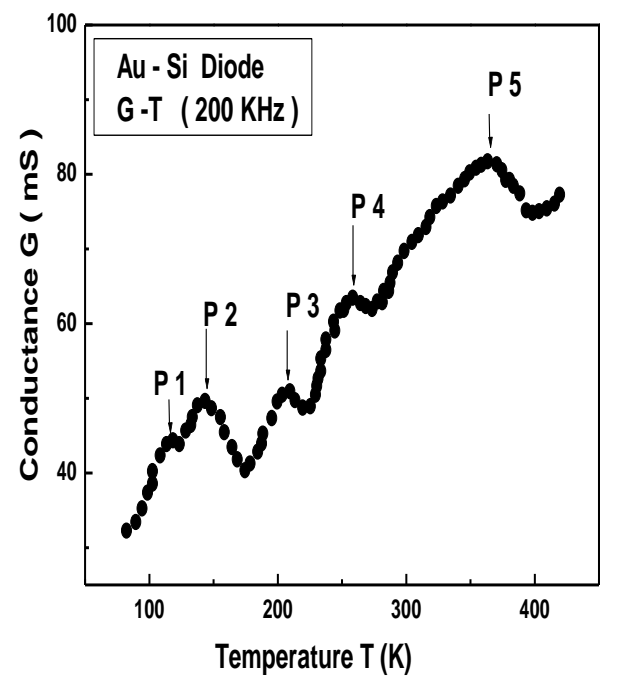

Fig. 5 - Example of a G-T curve obtained on Au- phosphorus doped polycrystalline silicon Schottky diode

or $C(f)$ are plotted at a fixed temperature $T$. The first curve presents a peak at a frequency $\mathrm{fm}$ in the case of a trap presence whereas the second shows a point of in- flection at the same frequency $f_{m}$. Obviously, the $G-T$ plots are preferred because first it is easier to find the temperature of a peak than a point of a curve inflection; second, also it is more simple to sweep the sample temperature than to regulate it at a fixed value. In Fig. 4, four curves $G(f)$ are given at indicted temperature. This figure shows the expected behaviour of the spectroscopy of a Schottky device that is an increase of the peak frequency $f_{m}$ when the temperature parameter increased, the same effect, with an increase of $T_{m}$ is observed if the $G-T$ spectroscopy is used.

An example of a $G-T$ curve is shown in Fig. 5 five peaks are highlighted in this curve. The temperature location of these peaks depends on the measurement frequency as previously claimed. Fig. 6 shows an example of an Arrhenius plot $e_{i} / T_{m i}{ }^{2}$ as a function of $1 / T_{m i}$ for the peak 5 using 4 frequency values. The slope of this plot gives the energy position of the trap, that $0.46 \mathrm{eV}$ here. Table 1 summarizes the energy positions corresponding to the 5 peak. The increasing energy and the close values for the 4 first peaks can indicate that these peaks correspond to a continuum that may be the conduction band tail. The fifth peak giving a higher energy of $0.46 \mathrm{eV}$ seems related to the Gaussian band usually found in disordered silicon. It may also relate to a gold diffusion that may occur from the metallic contact as previously mentioned. The lower density $N_{d}$ found near the interface from the $C$ - $V$ measurements can support this assertion. Future Au profile measurements have to be performed to check the Au diffusion.

Table 1 - Energy position corresponding to the 5 peaks of Fig. 5

\begin{tabular}{|l|l|l|l|l|l|}
\hline Peak & 1 & 2 & 3 & 4 & 5 \\
\hline Energy $(\mathrm{eV})$ & 0.016 & 0.026 & 0.030 & 0.13 & 0.46 \\
\hline
\end{tabular}

In any case, the example the spectroscopy admittance treatment presented here show the powerful possibilities to use this technique in the characterization of the electrical parameters of polysilicon films perpendicularly to their surface.

\section{CONCLUSION}

Au-polycrystalline silicon Schottky diodes are successfully made in this study. Their current-bias and capacitance bias characteristics gave evidence for their high quality with a high rectifying ratio, greater than $10^{4}$ at $+/-1 \mathrm{~V}$. The diodes are used to characterise the silicon material. The expected doping value was determined from the $C-V$ measurements. However low doping value was found near the interface. It was assumed due to the possible diffusion of $\mathrm{Au}$ atoms from the contacts or to oxygen diffusion from the ambience. Future $\mathrm{Au}$ profile measurements are needed. The possibility to use the $C-V$ measurements to obtain the doping profile shows that polycrystalline silicon is closer than crystalline compared to the amorphous material.

The known admittance spectroscopy technique was used to characterise the defect density in the upper half band gap. First measurements and analysis have shown the possibility to use this technique to check the conduction band tail as well as the deep defects. 


\section{REFERENCES}

1. S. Uchikoga, N. Ibaraki, Thin Solid Films 383, 19 (2001).

2. T. Matsuyama, M. Tanaka, S. Tusuda, S. Nakano, Y. Kuwano, Jpn. J. Appl. Phys. 32, 3720 (1993).

3. Wei Li, S. Varlamov, Chaowei Xue, Appl. Surf. Sci. 314, 174 (2014)

4. P. Zhang, E. Jacques, R. Rogel, O. Bonnaud, Solid-State Electron 86, 1 (2013).

5. G. Harbeke, L. Krausbauer, E.F. Steigmeier, A.E. Widmer, H.F. Kappert, G. Neugebauer, Appl. Phys. Lett. 42, 249 (1983).

6. S. Karatas, S. Altındal, A. Türüt, M. Cakar, Physica B 392 , 43 (2007).

7. Sandeep Kumar, Y.S. Katharria, Sugam Kumar, D. Kanjilal, Solid-Stat Electron. 50, 1835 (2006).

8. J.D. Cohen, D.V. Lang, Phys. Rev. B 25, 5321 (1982).
9. J.P. Kleider, P. Roca Cabarrocas, J. Non-Cryst. Solid. 299302, 599 (2002).

10. Nishant Chandra, Clarence J. Tracy, Jeong-Hyun Cho, S.T. Picraux, Raghuraj Hathwar, Stephen M. Goodnick, J. Appl. Phys. 118, 024301 (2015).

11. Ashish Kumar, Seema Vinayak, R. Singh, Curr. Appl. Phys. 13, 1137 (2013).

12. A. Keffous, M. Siad, A. Cheriet, N. Benrekaa, Y. Belkacem, Appl. Surf. Sci. 236, 42 (2004).

13. Mostefa Benhaliliba, J. Nano- Electron. Phys. 7 No 2, 02029 (2015)

14. D.L. Losee, J. Appl. Phys. 46, 2204 (1975).

15. O.S. Elsherif, K.D. Vernon-Parry, J.H. Evans-Freeman, R.J. Airey, M. Kappers, C.J. Humphreys, Physica B 407, 2960 (2012). 